\title{
Error dependent on renal function when monoexponential
}

\section{equation assumed}

\section{Serum clearances of ${ }^{125}$-iothalamate and ${ }^{131} \mid$-o-iodohippurate}

\begin{abstract}
An example from the literature has been used to demonstrate errors involved in calculating drug clearance by inappropriate use of the apparent drug distribution volume $V_{\mathrm{dext}}$. The $V_{\mathrm{dext}}$ is always an overestimate of the true volume of distribution in a multicompartment system, and the degree of overestimation in using it to calculate clearance for such a system will increase as renal function increases. Drug dosages calculated on the basis of overestimated clearance values may give rise to overdosage in normal individuals, or therapeutic failure in severely uremic patients. Problems associated with the use of an oversimplified pharmacokinetic model for clearance calculations are discussed, together with the concept of model-independent calculations.
\end{abstract}

\author{
Janis J. MacKichan, Pharm.D., Michael R. Dobrinska, M.S., \\ Peter G. Welling, Ph.D., and John G. Wagner, Ph.D. \\ Ann Arbor, Mich., and Madison, Wis. \\ College of Pharmacy and Upjohn Center for Clinical Pharmacology, the University of \\ Michigan, Ann Arbor, and the School of Pharmacy, University of Wisconsin, Madison
}

Drug clearance (sometimes called "metabolic clearance rate") is an important pharmacokinetic parameter useful in the evaluation of hepatic or renal function and adjustment of drug dosage regimens. It was first defined in 1956 by Hoenig and Schück ${ }^{3}$ as the ratio of intravenous dose to the area under the plasma concentration-time curve from zero to infinity, which has since been used to calculate the renal clearance of ${ }^{125}$ I-iothalamate, ${ }^{1}$ the plasma clearance of penicillin, ${ }^{10}$ and the metabolic clearance rates of cortisol ${ }^{5}$ and prednisolone. ${ }^{4}$

Although this formula is not based on the

Received for publication June 6, 1977

Accepted for publication July 8, 1977.

Reprint requests to: Dr. John G. Wagner, Upjohn Center for Clinical Pharmacology, University of Michigan Medical Center, Ann Arbor, Mich. 48109. assumption of a pharmacokinetic model, model-specific formulas have been derived. For the one-compartment open model, clearance is simplified to the product of $\lambda_{1}$ (the apparent first-order elimination rate constant) and $V_{d a p p}$ * (the apparent volume of distribution). Clearance for all other linear compartment models is equal to the product of $\lambda_{1}$ and $V_{\text {darea }}$ (the volume of distribution at any time during the terminal log-linear phase of drug elimination). (See Appendix, Equations 1 to 11 for derivations.)

Unfortunately, too many investigators inappropriately assume a one-compartment open model by using $V_{d a p p}$ or $V_{\text {dext }}$ to calculate clearance for data which are perhaps better fitted to a

\footnotetext{
$* \mathrm{~V}_{\text {dapp }}$ is often used interchangeably with $\mathrm{V}_{\text {dext }}$, the extrapolated volume of distribution.
} 
Table I. Summary of average pharmacokinetic parameters for $O I H^{131} \mathrm{I}$

\begin{tabular}{|c|c|c|c|}
\hline Parameter & Group I & Group 2 & Group 3 \\
\hline $\mathrm{C}_{\mathrm{Cl}} *(\mathrm{ml} / \mathrm{min})$ & $77.8(39.7) \dagger(50) \ddagger$ & $47.6(35.7)(17)$ & $18.5(30.5)(6)$ \\
\hline$\lambda_{1}\left(\mathrm{hr}^{-1}\right)$ & $0.46(41.9)(53)$ & $0.33(32.8)(19)$ & $0.22(32.7)(7)$ \\
\hline $\mathrm{V}_{\text {darea }}(\mathrm{L})$ & $60.6(71.9)(53)$ & $39.1(68.1)(19)$ & $31.8(34.2)(7)$ \\
\hline$V_{\text {dext }}(L)$ & $112(89.1)(53)$ & $61.4(99.3)(19)$ & $37.0(40.3)(7)$ \\
\hline $\begin{array}{r}V_{\text {dext }} \cdot \lambda_{1} \\
(\mathrm{ml} / \mathrm{min})\end{array}$ & $691(51.5)(53)$ & $272(48.3)(19)$ & $134(54.7)(7)$ \\
\hline $\begin{array}{r}\mathrm{V}_{\text {darea }} \cdot \lambda_{1} \\
(\mathrm{ml} / \mathrm{min})\end{array}$ & $399(47.3)(53)$ & $188(39.1)(19)$ & $114(47.1)(7)$ \\
\hline $\begin{array}{l}\text { Percentage } \\
\text { error }(\%)\end{array}$ & $74.7(85.1)(53)$ & $42.7(82.4)(19)$ & $14.8(67.4)(7)$ \\
\hline \multicolumn{4}{|c|}{$\begin{array}{l}\text { *Creatinine clearance. } \\
+ \text { Coefficient of variation }(\%): \frac{\text { standard deviation }}{\text { mean }} \times 100\end{array}$} \\
\hline Parameter & Group I & Group 2 & Group 3 \\
\hline $\mathrm{C}_{\mathrm{Cl}}(\mathrm{ml} / \mathrm{min})$ & $80.0(37.1)(45)$ & $45.1(38.8)(19)$ & $18.8(50.9)(8)$ \\
\hline$\lambda_{1}\left(\mathrm{hr}^{-1}\right)$ & $0.25(34.6)(46)$ & $0.15(39.2)(21)$ & $0.07(50.7)(8)$ \\
\hline $\mathrm{V}_{\text {darea }}(\mathrm{L})$ & $22.6(21.7)(46)$ & $19.6(37.4)(21)$ & $21.7(19.0)(8)$ \\
\hline $\mathrm{V}_{\mathrm{dext}}(\mathrm{L})$ & $26.1(27.7)(46)$ & $21.0(36.9)(21)$ & $22.3(18.7)(8)$ \\
\hline $\begin{array}{r}\mathrm{V}_{\text {dext }} \cdot \lambda_{1} \\
(\mathrm{ml} / \mathrm{min})\end{array}$ & $110(40.1)(46)$ & $49.4(42.2)(21$ & $26.8(57.0)(8)$ \\
\hline $\begin{array}{r}\mathrm{V}_{\text {darea }} \cdot \lambda_{1} \\
(\mathrm{ml} / \mathrm{min})\end{array}$ & $95.5(37.1)(46)$ & $45.8(39.7)(21)$ & $25.9(57.1)(8)$ \\
\hline $\begin{array}{l}\text { Percentage } \\
\text { error }(\%)\end{array}$ & $14.3(58.9)(46)$ & $7.19(90.6)(21)$ & $3.33(56.6)(8)$ \\
\hline
\end{tabular}

more complex model. Clearances have been calculated for intravenous antipyrine and tolbutamide $^{8}$ as well as oral pentobarbital ${ }^{7}$ using the formula specific for the one-compartment open model (Equation 7). Other workers, ${ }^{6,11,12}$ however, have shown that the data of these drugs are actually best described by a bioexponential equation and not by a monoexponential equation as the one-compartment model requires.

Although it has been shown that error is introduced when parameter estimates for multicompartment data are calculated by equations for the simple one-compartment system, ${ }^{9,}{ }^{14}$ the magnitude and significance of this error have not been demonstrated. This study was undertaken to quantitate the degree of error introduced when drug clearance is calculated by the use of an incorrect pharmacokinetic model. By purposely assuming a one-compartment model to calculate serum clearance for data described by a biexponential equation, we show not only the degree of error in the calculated clearance values, but, more importantly, the dependence of percentage error on renal function. The importance of model-independent pharmacokinetic parameter calculations is discussed.

\section{Methods}

The pharmacokinetic data of ${ }^{131} \mathrm{I}-\mathrm{o}-$ iodohippurate $\left(\mathrm{OIH}^{131} \mathrm{I}\right)$ and ${ }^{125}$ I-iothalamate (IOT $\left.{ }^{125} \mathrm{I}\right)$ from Welling and co-workers ${ }^{15}$ were used in this investigation. In the original study, male patients were divided into three groups on the basis of renal function: group 1 (53 patients) was designated as normal with serum creatinines equal to or less than $1.5 \mathrm{mg}$ per 100 $\mathrm{ml}$; group 2 (21 patients), with serum creatinines between 1.6 and $2.5 \mathrm{mg}$ per $100 \mathrm{ml}$, was designated moderately uremic; and group 3 

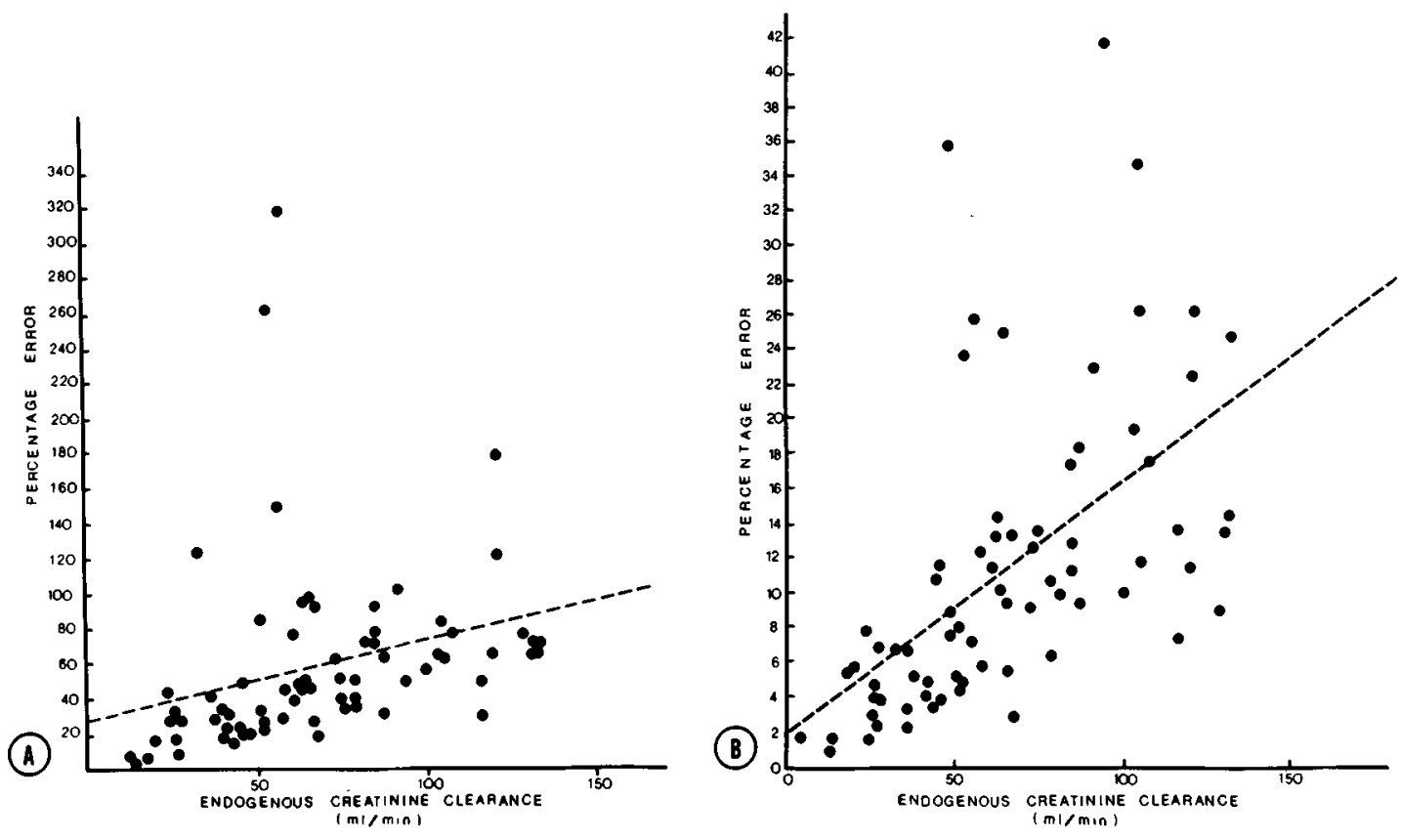

Fig. 1. Relationship of percentage error in clearance calculation to endogenous creatinine clearance for $\mathrm{OIH}^{131} \mathrm{I}(A)$ and $\mathrm{IO}^{125} \mathrm{I}(B)$, all patients. The dashed lines represent the least squares lines of best fit: $\mathrm{r}=0.28\left(\mathrm{OIH}_{131} \mathrm{I}\right)$ and $0.56\left(\mathrm{IOT}^{125} \mathrm{I}\right)$.

(9 patients) was designated severely uremic with serum creatinines greater than $2.5 \mathrm{mg}$ per $100 \mathrm{ml}$. Each patient received 10 to $20 \mu \mathrm{Ci}$ of $\mathrm{OIH}^{131} \mathrm{I}$ and $\mathrm{IOT}^{125} \mathrm{I}$ as indicators of renal function by rapid intravenous injection. Serum samples were obtained immediately before and at $0.25,0.5,1.0,2.0,4.0$, and $8.0 \mathrm{hr}$ following injection. In the present study the coefficients and exponents of the biexponential equation describing the serum concentration-time curve (Equation 3) were obtained enabling the calculation of $V_{\text {dext }}$ and $V_{\text {darea }}$ for each patient using Equations 8 and 10. Serum clearances were calculated two ways for each patient (Equations 7 and 9), and the percentage difference between the values was found by

$$
\frac{\left(\mathrm{V}_{\text {dext }} \cdot \lambda_{1}\right)-\left(\mathrm{V}_{\text {darea }} \cdot \lambda_{1}\right)}{\left(\mathrm{V}_{\text {darea }} \cdot \lambda_{1}\right)} \times 100
$$

where $V_{\text {darea }} \cdot \lambda_{1}$ is the true serum clearance and $\lambda_{1}$ is the smallest rate constant. The means, standard deviations, and coefficients of variation were calculated for all parameters, and linear regression analysis was done to relate percentage error in clearance calculation to the endogenous creatinine clearance.

\section{Results}

The mean pharmacokinetic parameters on $\mathrm{OIH}^{131} \mathrm{I}$ and IOT $^{125} \mathrm{I}$ are given in Tables I and II. Included in the tables are the endogenous creatinine clearance and the percentage error introduced by drug clearance calculations based on the assumption of a one-compartment model. With both drugs, the mean percentage error was large in normal patients, less in patients with moderate uremia, and quite small in severely uremic individuals. The relationship of percentage error in calculation of drug clearances to the endogenous creatinine clearance is illustrated in Fig. 1. Fig. 2 graphically depicts the effect of renal impairment, represented by the three patient groups, on the mean percentage error. The difference among the mean percentage errors for the three groups was significant $(p<0.005)$ for both drugs, using the Kruskal-Wallis $\mathrm{H}$ test. $^{2}$

\section{Discussion}

Wagner and Northam ${ }^{14}$ have shown that the apparent or extrapolated volume of distribution in a multicompartment open system is always an overestimate of the true total volume. It fol- 

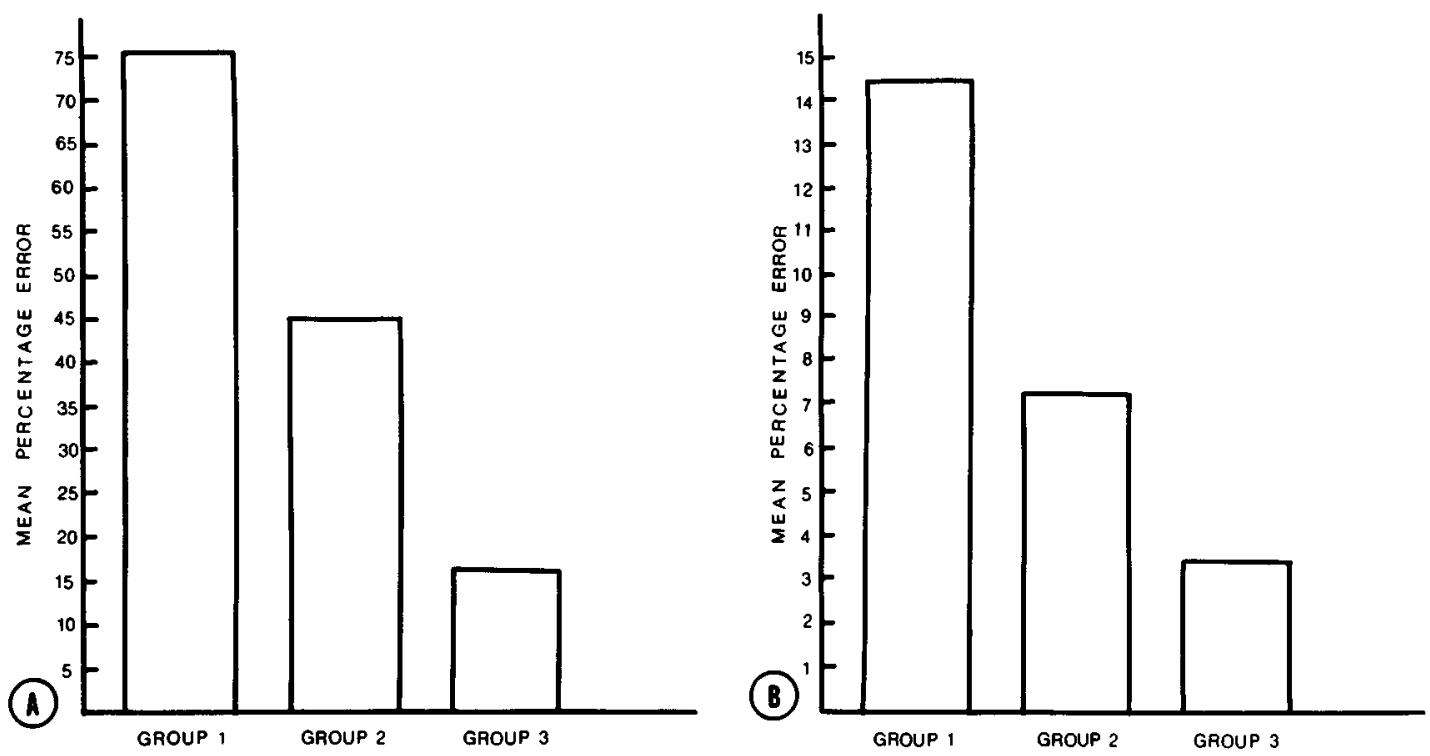

Fig. 2. Effect of degree of renal impairment on mean percentage error in clearance calculation for $\mathrm{OIH}^{131} \mathrm{I}(A)$ and IOT $^{125} \mathrm{I}(B)$. Group I, normal; Group 2, moderately uremic; Group 3, severely uremic.

lows that clearances calculated using $\mathrm{V}_{\text {dext }}$ for data described by a biexponential equation will also be overestimated as verified by our results, where the mean overestimation for $\mathrm{OIH}^{131}$ I was as high as $75 \%$. Equation 13 in the appendix shows that the degree of error introduced depends on the relative values of the coefficients and exponents of the biexponential equation describing the system, or it depends on how "two-compartment" the system is. In renal failure, the elimination rate constant, $\lambda_{1}$, is smaller, thus decreasing the ratio indicative of degree of error. This is demonstrated in Figs. 1 and 2 where the percentage error is seen to decrease as renal function decreases.

The clinical importance of this error cannot be overlooked. Because the magnitude of error may be large, and because it depends on the degree of renal function, the use of the onecompartment formula for data fitted by a biexponential equation may be unreliable, especially for drugs excreted primarily by the kidney. As overestimation of clearance increases with increasing renal function, the use of $\mathrm{V}_{\mathrm{dext}}$ could give rise to drug overdoses in normal individuals. Alternatively, if the calculated dose produces a required therapeutic effect in normals, dose adjustment in uremic patients based on clearance values using $\mathrm{V}_{\text {dext }}$ may lead to therapeutic failure due to disproportionate dose reduction. If serum or plasma clearances are used to evaluate renal function, as for $\mathrm{OIH}^{131} \mathrm{I}$ and IOT $^{125}$, overestimation of the clearances will indicate that the renal function is better than it actually is. In general, because plasma or serum clearance is often used to adjust drug dosage and evaluate renal function, a large degree of error in its calculation may adversely affect a patient's therapeutic regimen.

Because the assumption of an incorrect pharmacokinetic model for a set of blood concentration vs time data may lead to a high degree of error in clearance calculations, an alternative method for these calculations might be used. Due to the uncertainty of the correctness of a particular pharmacokinetic model, another approach to the problem would be one that is "model-independent." Instead of assuming a model and basing parameter calculations on that model, one can find the polyexponential equation that best fits the experimental data and calculate clearance directly from the coefficients and exponents of that equation. ${ }^{13}$ Errors associated with assumption of an incorrect pharmacokinetic model may be eliminated in this manner. 


\section{References}

1. Adefuin, P. Y., Gur, A., Siegel, N. J., Spencer, R. P., and Hayslett, J. P.: Single subcutaneous injection of Iothalamate Sodium $I^{125}$ to measure glomerular filtration rate, J. A. M. A. 235: $1467-1469,1976$.

2. Beyer, W. H., editor: CRC Handbook of tables for probability and statistics, Cleveland, Ohio, 1966, The Chemical Rubber Co., p. 326.

3. Hoenig, V., and Schück, O.: Hepatic clearance theory and clinical application, Rev. Czech. Med. 2:1-14, 1956.

4. Kozower, M., Veatch, L., and Kaplan, M. M.: Decreased clearance of prednisolone, a factor in the development of corticosteroid side effects, $\mathrm{J}$. Clin. Endocrinol. Metab. 38:407-412, 1974.

5. deLacerda, L., Kowarski, A., and Migeon, C. J.: Diurnal variation of the metabolic clearance rate of cortisol. Effect of measurement of cortisol production rate, J. Clin. Endocrinol. Metab. 36: 1043-1049, 1973.

6. Matin, S. B., Wan, S. H., and Karam, J. H.: Pharmacokinetics of tolbutamide: Prediction by concentration in saliva, Clin. Pharmacol. THER. 16:1052-1058, 1974.

7. Reidenberg, M. M., Lowenthal, D. T., Briggs, W., and Gasparo, M.: Pentobarbital elimination in patients with poor renal function, CLIN. Pharmacol. Ther. 20:67-71, 1976.

8. Reidenberg, M. M., and Vesell, E. S.: Unaltered metabolism of antipyrine and tolbutamide in fasting man, Clin. Pharmacol. Ther. 17:650-656, 1975.
9. Riegelman, S., Loo, J. C. K., and Rowland, M.: Shortcomings in pharmacokinetic analysis by conceiving the body to exhibit properties of a single compartment, J. Pharm. Sci. 57:117-123, 1968.

10. Schück, O., and Šmahel, O.: Plasma clearance of some penicillin preparations, Antibiot. Med. Clin. Ther. 5:98-103, 1958.

11. Smith, R. B., Dittert, L. W., Girffen, W. O., and Doluisio, J. T.: Pharmacokinetics of pentobarbital after intravenous and oral administration, J. Pharmacokinet. Biopharm. 1:5-16, 1973.

12. Swartz, R. D., Sidell, F. R., and Cucinell, S. A.: Effects of physical stress on the disposition of drugs eliminated by the liver in man, $\mathrm{J}$. Pharmacol. Exp. Ther. 188:1-7, 1974.

13. Wagner, J. G.: Linear pharmacokinetic equations allowing direct calculation of many needed pharmacokinetic parameters from the coefficients and exponents of polyexponential equations which have been fitted to the data, J. Pharmacokinet. Biopharm. 4:443-467, 1976.

14. Wagner, J. G., and Northam, J. I.: Estimation of volume of distribution and half-life of a compound after rapid intravenous injection, $J$. Pharm. Sci. 56:529-531, 1967.

15. Welling, P. G., Mosegaard, A., Dobrinska, M. R., and Madsen, P. O.: Pharmacokinetics of ${ }^{125}$ I-iothalamate and ${ }^{131}$ I-o-iodohippurate in man, J. Clin. Pharmacol. 16:142-148, 1976.

\section{Appendix}

The general polyexponential equation describing a plasma or serum concentration versus time curve is

$$
\mathrm{C}^{\mathrm{IV}}=\Sigma \mathrm{C}_{\mathrm{i}} \mathrm{e}^{-\lambda_{\mathrm{i}} \mathrm{t}}
$$

where $\mathrm{C}^{\mathrm{IV}}$ is the concentration in plasma or serum following bolus intravenous injection.

The monoexponential equation

$$
\mathrm{C}^{\mathrm{IV}}=\mathrm{C}_{1} \mathrm{e}^{-\lambda_{1} \mathrm{t}}
$$

describes the classic one-compartment open model, while the biexponential equation

$$
\mathrm{C}^{\mathrm{IV}}=\mathrm{C}_{1} \mathrm{e}^{-\lambda_{2} \mathrm{t}}+\mathrm{C}_{2} \mathrm{e}^{-\lambda_{2} \mathrm{t}}
$$

describes the classic two-compartment open model.

The general equation for finding the area under the concentration-time curve from zero to infinity is simply the sum of the ratio of coefficients to their exponents:

$$
\mathrm{AUC}_{0 \rightarrow \infty}=\Sigma \frac{\mathrm{C}_{1}}{\lambda_{\mathrm{i}}}
$$

which for a one-compartment open model is equal to

$$
\mathrm{AUC}_{0 \rightarrow \infty}=\frac{\mathrm{C}_{1}}{\lambda_{1}}
$$

and for a two-compartment open model equals

$$
\mathrm{AUC}_{0 \rightarrow \infty}=\frac{\mathrm{C}_{1}}{\lambda_{1}}+\frac{\mathrm{C}_{2}}{\lambda_{2}}
$$


Thus, the clearances for each model, defined as the ratio of the intravenous dose to the area under the concentration-time curve from zero to infinity, are given by Equations 7 and 9 .

One compartment model:

$$
\begin{gathered}
\mathrm{Cl}=\frac{\text { Dose }^{\mathrm{IV}}}{\mathrm{AUC}_{0 \rightarrow \infty}}=\frac{\text { Dose }^{\mathrm{IV}}}{\mathrm{C}_{1} / \lambda_{1}}=\mathrm{V}_{\text {dext }} \cdot \lambda_{1} \\
\text { where } \mathrm{V}_{\text {dext }}=\frac{\text { Dose }^{\mathrm{IV}}}{\mathrm{C}_{1}} .
\end{gathered}
$$

Multicompartment model:

$$
\mathrm{Cl}=\frac{\text { Dose }^{\mathrm{IV}}}{\mathrm{AUC}_{0 \rightarrow \infty}}=\frac{\text { Dose }^{\mathrm{IV}}}{\Sigma \frac{\mathrm{C}_{\mathrm{i}}}{\lambda_{\mathrm{i}}}}=\mathrm{V}_{\mathrm{darea}} \cdot \lambda_{\mathrm{i}}
$$

where

$$
\mathrm{V}_{\text {darea }}=\frac{\text { Dose }^{\mathrm{IV}}}{\lambda_{1} \Sigma\left(\mathrm{C}_{\mathrm{i}} / \lambda_{\mathrm{i}}\right)}
$$

and for data described by a biexponential equation:

$$
\mathrm{V}_{\text {darea }}=\frac{\text { Dose }^{\mathrm{IV}}}{\lambda_{1}\left(\mathrm{C}_{1} / \lambda_{1}+\mathrm{C}_{2} / \lambda_{2}\right)}
$$

One can see that the clearance formula for a one-compartment model cannot be applied to data described by anything except a monoexponential equation. If a one-compartment model is assumed for polyexponential data, the ratio of clearance calculated by $\left(V_{\text {dext }} \cdot \lambda_{1}\right)$ to the true clearance $\left(V_{\text {darea }} \cdot \lambda_{1}\right)$ is given by:

$$
\frac{\mathrm{V}_{\text {dext }} \cdot \lambda_{1}}{\mathrm{~V}_{\text {darea }} \cdot \lambda_{1}}=\frac{\mathrm{V}_{\text {dext }}}{\mathrm{V}_{\text {darea }}}=\frac{\frac{\text { Dose }^{\mathrm{IV}} / \mathrm{C}_{1}}{\text { Dose }^{\mathrm{IV}}}}{\lambda_{1}\left(\Sigma \mathrm{C}_{\mathrm{i}} / \lambda_{\mathrm{i}}\right)}
$$

When a one-compartment model is erroneously assumed for data described by a biexponential equation, this ratio is equal to:

$$
\frac{\frac{\text { Dose }^{\mathrm{IV}} / \mathrm{C}_{1}}{\text { Dose }^{\mathrm{IV}}}}{\lambda_{1}\left(\mathrm{C}_{1} / \lambda_{1}+\mathrm{C}_{2} / \lambda_{2}\right)}=1+\frac{\mathrm{C}_{2} \lambda_{1}}{\mathrm{C}_{1} \lambda_{2}} .
$$

Thus, it can be seen that the error in calculation of clearance by assumption of a one-compartment open model depends on the values of both coefficients $C_{1}$ and $C_{2}$, and both rate constants, $\lambda_{1}$ and $\lambda_{2}$. 